\title{
Mathematical modeling of elastic disturbance propagation in a structure containing a porous layer saturated with gas and water
}

\author{
Chekurin V. ${ }^{1,2}$, Pavlova A. ${ }^{3}$ \\ ${ }^{1}$ Pidstryhach Institute for Applied Problems of Mechanics and Mathematics of NAS of Ukraine \\ 3-b Naukova str., 79060, Lviv, Ukraine \\ ${ }^{2}$ Kuyawy and Pomorze University in Bydgoszcz \\ 55-57 Torunska str., 85-023 Bydgoszcz, Poland \\ ${ }^{3}$ Carpathian Branch of Subbotin Institute of Geophysics of NAS of Ukraine \\ 3-b Naukova str., 79060, Lviv, Ukraine
}

(Received 1 October 2016)

\begin{abstract}
A horizontally layered elastic structure containing a homogeneous porous layer saturated partly with gas and partly with water is considered. The paper is aimed at studying of interaction of elastic waves, caused by local pulse source, with the structure. The boundary-value problem describing the wave dynamics of the structure is formulated. A mathematical model describing distributions of the gas and water in a pore space of the porous layer depending of the amount of the gas accumulated in the layer is developed. The problem is solved with the use of Fourier transform. It was established that wavefield pattern on the free surface of the structure is dependent on amount of gas accumulated in the porous layer. Quantitative measures relating the wavefield parameters on the structure's free surface and the amount of gas accumulated in the porous layer are introduced. The obtained results can be used to develop distance methods for accounting of amount of natural gas accumulated in underground gas storage facilities built in aquifers.
\end{abstract}

Keywords: layered structure, porous media, elastic disturbance.

2000 MSC: $74 \mathrm{~J} 20$

UDC: 550.344

\section{Introduction}

Porous stratums of geological structures are often used for construction of underground natural gas storage facilities [1]. If the storage is developed in an aquifer (a porous medium saturated with water), the stratum is being saturated partly with gas and partly by water in its operational state. When the storage is being filled or emptied, the boundary dividing its parts saturated with gas and water is displacing along the vertical coordinate. As the elastic moduli of saturated porous media are dependent on physical properties and thermodynamic state of the saturants, the porous layer should be considered as a heterogeneous two-layer structure. The thicknesses of the layers and their elastic properties are changed depending on amount of the gas accumulated in the layer. This can be used for developing of distance methods for accounting of the gas accumulated in the layer with the use of acoustic methods. So we come to the necessity of mathematical modeling and quantitative studying the interaction of elastic waves with layered structures containing porous layers saturated with gas and water.

There are various aspects in studying of elastic waves in porous media - modeling of dynamics, dispersion analysis of wave motion, wave velocity and attenuation, interference of elastic waves etc.

An approach, which is effectively used to describe dynamics of porous medium, is based on homogenization of microscopically heterogeneous body. Within this approach Gassmann derived relations [2], which express the effective elastic properties of the porous saturated medium via the individual elastic properties of the solid skeleton and the saturant. This approach is valid for sufficiently low frequencies of wave motion, when mutual displacement of the skeleton and the fluid is negligible. Such, for instance, are seismic waves arising in geological structure [3-5]. 
Many authors studied wave-field pattern in layered structures. Particular interest in these researches exists in geophysics, where the objects of study are layered rock structures [6]. The interest is stimulated by practical problems of acoustic logging, detecting and identification of seismic sources, prediction of tectonic earthquakes etc. To solve such problems in the case of piece-wise homogeneous horizontallylayered structure so called matrix method was developed [7,8]. By applying integral transforms, this method enables to reduce the problem of interaction of elastic waves with the $n$-layered structure to boundary value problem for $n$ systems of ordinary differential equations. The systems are coupled via contact conditions acting on the boundaries which divide adjacent layers.

In spite of large number of publications devoted to problems of wave dynamics in application to geophysics of layered structures and wide utilization of the matrix method for solving of such problems, the problems of interaction of elastic wave with layered structure containing porous layers saturated with different fluids remain unstudied. In this paper we consider such a problem.

This paper is aimed on studying of interaction of elastic disturbances, caused by local pulse source, with a semi-infinite layered structure containing a porous stratum saturated with gas and water. We study patterns of wave interference on the free surface of the structure depending on the position of gas-water interface in the porous layer. Obtained results can be used to develop distance methods for accounting of amount of natural gas accumulated in underground gas storage facilities build in aquifers.

\section{Problem formulation}

We consider the semi-infinite layered structure, consisting of three infinite elastic layers $\mathrm{E}_{I}$ to $\mathrm{E}_{I I I}$ and elastic half-space $\mathrm{E}_{I V}$ :

$$
\begin{gathered}
\mathrm{Ł}_{I}=\left\{-\infty<x_{1}<\infty,-\infty<x_{2}<\infty, 0<x_{3}<L-H\right\}, \\
\mathrm{Ł}_{I I}=\left\{-\infty<x_{1}<\infty,-\infty<x_{2}<\infty, L-H<x_{3}<L-H+h\right\}, \\
\mathrm{Ł}_{I I I}=\left\{-\infty<x_{1}<\infty,-\infty<x_{2}<\infty, L-H<x_{3}<L-H+h\right\}, \\
\mathrm{E}_{I V}=\left\{-\infty<x_{1}<\infty,-\infty<x_{2}<\infty, L<x_{3}<\infty\right\} .
\end{gathered}
$$

Here $x_{i}, i=1,2,3$ stand for Cartesian coordinates, $L>0$ is the depth of the porous stratum, $H>L$ stands for its thickness, $h \in[0, H]$ is the thickness of the layer saturated with gas.

Wave field in each layer satisfies three groups of relations of the theory of elasticity [9]: equations of motion

$$
\rho_{\alpha} \frac{\partial^{2} u_{i}^{\alpha}}{\partial t^{2}}=\frac{\partial \sigma_{i j}^{\alpha}}{\partial x_{j}}+\rho^{\alpha} f_{i}^{\alpha}, \quad j=1,2,3
$$

elasticity relations

$$
\sigma_{i j}^{\alpha}=2 \mu_{\alpha} e_{i j}^{\alpha}+\lambda_{\alpha} e_{k l}^{\alpha} \delta_{k l} \delta_{i j},
$$

and compatibility relations (in Cauchy form)

$$
e_{i j}^{\alpha}=\frac{1}{2}\left(\frac{\partial u_{i}^{\alpha}}{\partial x_{i}}+\frac{\partial u_{j}^{\alpha}}{\partial x_{j}}\right) .
$$

Here $\rho_{\alpha}$ is mass density of elastic medium in $\alpha$-layer $(\alpha=I, I I, I I I, I V), u_{i}^{\alpha}, \sigma_{i j}^{\alpha}, e_{i j}^{\alpha}$ stand for Cartesian components of displacement vector, stress tensor, and strain tensor correspondingly of $\alpha$-layer, $\lambda_{\alpha}$ and $\mu_{\alpha}$ are elastic moduli (Lame coefficients) $f_{i}^{\alpha}=f_{i}^{\alpha}\left(x_{1}, x_{2}, x_{3}, t\right)$ stand for $i$-th Cartesian component of body force in $\alpha$-layer.

With the use of the relations (2) and (3) the equation (1) can be reduced to the hyperbolic system of equations

$$
\rho_{\alpha} \frac{\partial^{2} u_{i}^{\alpha}}{\partial t^{2}}=\mu_{\alpha} \frac{\partial^{2} u_{i}^{\alpha}}{\partial x_{k} \partial x_{l}} \cdot \delta_{k l}+\left(\lambda_{\alpha}+\mu_{\alpha}\right) \frac{\partial}{\partial x_{i}} \frac{\partial u_{k}^{\alpha}}{\partial x_{l}} \cdot \delta_{k l}+f_{i}^{\alpha} .
$$

Mathematical Modeling and Computing, Vol.3, No. 2, pp. 120-134 (2016) 
The surface $x_{3}=0$ is free of traction

$$
\left.\sigma_{31}^{I}\right|_{x_{3}=0}=0,\left.\quad \sigma_{32}^{I}\right|_{x_{3}=0}=0,\left.\quad \sigma_{32}^{I}\right|_{x_{3}=0}=0 .
$$

We consider the interfaces between the layers as the boundary of ideal elastic contact, what can written as

$$
\begin{array}{ll}
\left.u_{i}^{I}\right|_{x_{3}=L-H}=\left.u_{i}^{I I}\right|_{x_{3}=L-H}, & \left.\sigma_{3 i}^{I}\right|_{x_{3}=L-H}=\left.\sigma_{3 i}^{I I}\right|_{x_{3}=L-H}, \\
\left.u_{i}^{I I}\right|_{x_{3}=L-H+h}=\left.u_{i}^{I I I}\right|_{x_{3}=L-H+h}, & \left.\sigma_{3 i}^{I I}\right|_{x_{3}=L-H+h}=\left.\sigma_{3 i}^{I I I}\right|_{x_{3}=L-H+h}, \\
\left.u_{i}^{I I I}\right|_{x_{3}=L}=\left.u_{i}^{I V}\right|_{x_{3}=L}, & \left.\sigma_{3 i}^{I I I}\right|_{x_{3}=L}=\left.\sigma_{3 i}^{I V}\right|_{x_{3}=L} .
\end{array}
$$

According to the relations (2), (3) the stress components $\sigma_{3 i}^{\alpha}$ in the relations (6)-(8) are considered and the function of displacement components $u_{i}^{\alpha}$.

We suppose additionally that the function $u_{i}^{\alpha}=u_{i}^{\alpha}\left(x_{1}, x_{2}, x_{3}, t\right)$ satisfies Sommerfeld radiation condition [10] at infinitely remote points. When the body forces $f_{i}^{\alpha}=f_{i}^{\alpha}\left(x_{1}, x_{2}, x_{3}, t\right)$ are given as a function of spatial coordinates and time, the problem (4)-(8) describes the wave field in the structure.

\section{The method for solving the problem}

Let there be an only source, which is localized in the layer $\mathrm{E}_{I}$ at the point $\left(x_{1}^{s}, x_{2}^{s}, x_{3}^{s}\right)$, where $x_{3}^{s} \in[0, L-H]$ :

$$
f_{I}^{I}=\varphi(t) \delta\left(x_{1}-x_{1}^{s}\right) \delta\left(x_{2}-x_{2}^{s}\right) \delta\left(x_{3}-x_{3}^{s}\right), \quad f_{i}^{\alpha}=0, \quad \alpha=I I, I I I, I V .
$$

Here $\varphi(t)$ is a function determining dependence in time of the source.

Now we can replace the equations (1) with corresponding homogeneous equations in each layer. To do that we will consider the layer $L_{I}$ as the union of two layers $\mathrm{E}_{I}=\mathrm{E}_{I^{\prime}} \bigcup \mathrm{E}_{I I^{\prime}}$, where

$$
\begin{gathered}
\mathrm{E}_{I^{\prime}}=\left\{-\infty<x_{1}<\infty,-\infty<x_{2}<\infty, 0<x_{3}<x_{3}^{s}\right\}, \\
\mathrm{E}_{I^{\prime \prime}}=\left\{-\infty<x_{1}<\infty,-\infty<x_{2}<\infty, x_{3}^{s}<x_{3}<L-H\right\} .
\end{gathered}
$$

Now the homogeneous equation of motion acts in each layer $\alpha=I^{\prime}, I^{\prime \prime}, I I, I I I, I V$

$$
\rho_{\alpha} \frac{\partial^{2} u_{i}^{\alpha}}{\partial t^{2}}-\frac{\partial \sigma_{i j}^{\alpha}}{\partial x_{j}}=0, \quad j=1,2,3
$$

The elasticity moduli of layers $\mathrm{E}_{I^{\prime}}$ and $\mathrm{E}_{I^{\prime \prime}}$ are defined as

$$
\lambda_{I^{\prime}}=\lambda_{I^{\prime \prime}}=\lambda_{I}, \mu_{I^{\prime}}=\mu_{I^{\prime \prime}}=\mu_{I} .
$$

To take into account the source, acting in the interface between $\mathrm{E}_{I^{\prime}}$ and $\mathrm{E}_{I^{\prime \prime}}$, we present the contact conditions on the boundary $x_{3}=x_{3}^{s}$ in the form

$$
\left.u_{i}^{I^{\prime}}\right|_{x_{3}=x_{3}^{s}}=\left.u_{i}^{I^{\prime \prime}}\right|_{x_{3}=x_{3}^{s}}=u_{i}^{s},\left.\quad \sigma_{3 i}^{I^{\prime}}\right|_{x_{3}=x_{3}^{s}}=\left.\sigma_{3 i}^{I^{\prime \prime}}\right|_{x_{3}=x_{3}^{s}}=\sigma_{i}^{s} .
$$

Now instead of relations (6) we have

$$
\left.u_{i}^{I^{\prime}}\right|_{x_{3}=L-H}=\left.u_{i}^{I I}\right|_{x_{3}=L-H},\left.\quad \sigma_{3 i}^{I^{\prime}}\right|_{x_{3}=L-H}=\left.\sigma_{3 i}^{I I}\right|_{x_{3}=L-H} .
$$

We will consider pulse sources $f_{i}^{\alpha}$ which varying in time like a peak shape function, for instance, like Gaussian $\varphi(t)=\exp \left(\left(t-t_{0}\right)^{2} / \tau_{0}^{2}\right)$. This enables us to apply the Fourier transform to them. 
Substituting relation (3) into (2), we obtain

$$
\mu_{\alpha}\left(\frac{\partial u_{i}^{\alpha}}{\partial x_{j}}+\frac{\partial u_{j}^{\alpha}}{\partial x_{i}}\right)+\lambda_{\alpha} \delta_{i j} \sum_{k=1}^{3} \frac{\partial u_{k}^{\alpha}}{\partial x_{k}}-\sigma_{i j}^{\alpha}=0
$$

So, we have the system (10), (13) of partial differential equations with respect to three component $u_{i}^{\alpha}$ of displacement vector and 6 components of stress tensor $\sigma_{i j}^{\alpha}$ (with accounting of its symmetry).

Under taken assumptions about behavior of the functions $u_{i}^{\alpha}\left(x_{1}, x_{2}, x_{3}, t\right), \sigma_{i j}^{\alpha}\left(x_{1}, x_{2}, x_{3}, t\right)$ and at infinity when $x_{1} \rightarrow \pm \infty$ and $x_{2} \rightarrow \pm \infty$ we can apply Fourier transform to them with respect to special coordinate $x_{1}$ and $x_{2}$. The structure (9) of the source enables to use this integral transform to the functions $u_{i}^{s}\left(x_{1}, x_{2}, x_{3}, t\right)$ and $\sigma_{i}^{s}\left(x_{1}, x_{2}, x_{3}, t\right)$. And finally, the assumption regarding the function $f(t)$ enables us to apply the Fourier transform with respect to time to all these functions.

Applying to equations (10) and (13) integral Fourier transform with respect to variables $x_{1}, x_{2}$ and $t$, we can reduce them to the system of 6 ordinary differential equations in independent variable $x_{3}$ with respect to variables $U_{1}^{\alpha}, U_{2}^{\alpha}, U_{3}^{\alpha}, S_{1}^{\alpha}, S_{2}^{\alpha}, S_{3}^{\alpha}$. The dependent variables are Fourier transforms of the functions $u_{1}^{\alpha}, u_{2}^{\alpha}, u_{3}^{\alpha}, \sigma_{31}^{\alpha}, \sigma_{32}^{\alpha}, \sigma_{33}^{\alpha}$ correspondingly:

$$
U_{i}^{\alpha} \equiv \mathcal{F}\left(u_{i}^{\alpha}\right), \quad S_{i}^{\alpha} \equiv \mathcal{F}\left(\sigma_{3 i}^{\alpha}\right) .
$$

Here

$$
\mathcal{F}(\ldots) \equiv \int_{-\infty}^{\infty} \int_{-\infty}^{\infty} \int_{-\infty}^{\infty} \exp \left\{-j \omega\left(t-x_{1} p_{1}-x_{2} p_{2}\right)\right\} \mathrm{d} x_{1} \mathrm{~d} x_{2} \mathrm{~d} t
$$

where $j \equiv \sqrt{-1}$ and $p_{1}, p_{2}$ are parameters of Fourier transforms with respect to time $t$ and special variables $x_{1}, x_{2}$.

We can write the system in matrix form

$$
\frac{\mathrm{d} \mathbf{B}_{\alpha}}{\mathrm{d} x_{3}}=j \omega \mathbf{A}_{\alpha} \cdot \mathbf{B}_{\alpha}
$$

Here $\mathbf{B}_{\alpha}$ stands for column-vector $6 \times 1 \mathbf{B}_{\alpha}=\left(\mathbf{U}_{\alpha}, \mathbf{S}_{\alpha}\right)^{\mathrm{T}}$ where $\mathbf{U}_{\alpha}=\left(U_{1}^{\alpha}, U_{2}^{\alpha}, U_{3}^{\alpha}\right)^{\mathrm{T}}, \mathbf{S}_{\alpha}=$ $(-j \omega)^{-1}\left(S_{1}^{\alpha}, S_{2}^{\alpha}, S_{3}^{\alpha}\right)^{\mathrm{T}}, A^{\alpha}$ is $6 \times 6$-matrix

$$
\mathbf{A}_{\alpha}=\left(\begin{array}{cc}
\mathbf{A}_{11}^{\alpha} & \mathbf{A}_{12}^{\alpha} \\
\mathbf{A}_{21}^{\alpha} & \mathbf{A}_{22}^{\alpha}
\end{array}\right)
$$

where

$$
\begin{gathered}
\mathbf{A}_{\mathbf{1 1}}^{\alpha}=\left(\begin{array}{ccc}
0 & 0 & p_{1} \\
0 & 0 & p_{2} \\
\frac{\lambda_{\alpha} p_{1}}{\lambda_{\alpha}+2 \mu_{\alpha}} & \frac{\lambda_{\alpha} p_{2}}{\lambda_{\alpha}+2 \mu_{\alpha}} & 0
\end{array}\right), \quad \mathbf{A}_{\mathbf{1 2}}^{\alpha}=\left(\begin{array}{ccc}
\frac{1}{\mu_{\alpha}} & 0 & 0 \\
0 & \frac{1}{\mu_{\alpha}} & 0 \\
0 & 0 & \frac{1}{\lambda_{\alpha}+2 \mu_{\alpha}}
\end{array}\right), \quad \mathbf{A}_{\mathbf{2 2}}^{\alpha}=\left(\mathbf{A}_{\mathbf{1 1}}{ }^{\alpha}\right)^{\mathrm{T}}, \\
\mathbf{A}_{\mathbf{2 1}}^{\alpha}=\left(\begin{array}{ccc}
-p_{1}^{2}\left(\lambda_{\alpha}+3 \mu_{\alpha}-\frac{\lambda_{\alpha}^{2}}{\lambda_{\alpha}+2 \mu_{\alpha}}\right)+\rho_{\alpha} & -p_{1} p_{2}\left(\lambda_{\alpha}+3 \mu_{\alpha}-\frac{\lambda_{\alpha}^{2}}{\lambda_{\alpha}+2 \mu_{\alpha}}\right)+\rho_{\alpha} & 0 \\
-p_{1} p_{2}\left(\lambda_{\alpha}+3 \mu_{\alpha}-\frac{\lambda_{\alpha}^{2}}{\lambda_{\alpha}+2 \mu_{\alpha}}\right)+\rho_{\alpha} & -p_{2}^{2}\left(\lambda_{\alpha}+3 \mu_{\alpha}-\frac{\lambda_{\alpha}^{2}}{\lambda_{\alpha}+2 \mu_{\alpha}}\right)+\rho_{\alpha} & 0 \\
0 & 0 & \rho_{\alpha}
\end{array}\right),
\end{gathered}
$$

As (16) is a system of ordinary differential equations with constant coefficients, its general solution can be presented via matrix exponential $[11,12]$

$$
\mathbf{B}_{\alpha}=\exp \left(j \omega\left(x_{3}-x_{0}\right) \mathbf{A}_{\alpha}\right) \cdot \mathbf{B}_{\alpha}^{\mathbf{0}} .
$$


The matrix exponential can be easily calculated by reducing the matrix $\mathbf{A}_{\alpha}$ to normal Jordan form [13]

$$
\mathbf{A}_{\alpha}=\mathbf{T}_{\alpha} \cdot \mathbf{J}_{\alpha} \cdot \mathbf{T}_{\alpha}^{-1} .
$$

Here $\mathbf{J}_{\alpha}$ is the normal Jordan form of the matrix $\mathbf{A}_{\alpha}, \mathbf{T}_{\alpha}$ is non-singular matrix. $\mathbf{J}_{\alpha}$ is an upper triangular quasidiagonal matrix: $\mathbf{J}_{\alpha}=\operatorname{diag}\left(\mathbf{J}_{\mathbf{0}}^{\alpha}, \mathbf{J}_{\mathbf{1}}^{\alpha}, \cdots, \cdots, \mathbf{J}_{\mathbf{K}}^{\alpha}\right)$. Block $\mathbf{J}_{\mathbf{0}}^{\alpha}$ is the diagonal matrix built of distinct eigenvalues $\lambda_{1}^{\alpha}, \lambda_{2}^{\alpha}, \cdots, \lambda_{M}^{\alpha}$ of the matrix $\mathbf{A}_{\alpha}: \mathbf{J}_{\mathbf{0}}^{\alpha}=\operatorname{diag}\left(\lambda_{1}^{\alpha}, \lambda_{2}^{\alpha}, \cdots, \lambda_{M}^{\alpha}\right)$, where $M \in \mathbb{N}$ is the number of distinct eigenvalues (real and complex). Each block $\mathbf{J}_{\mathbf{k}}^{\alpha}, k=1,2, \cdots, K$ corresponds to a repeated eigenvalue $\lambda_{k}^{\alpha}$. If $r_{k} \in \mathbb{N}$ is the number of multiplicity of the eigenvalue $\lambda_{k}^{\alpha}$, then $\mathbf{J}_{\mathbf{k}}^{\alpha}$ is $r_{k} \times r_{k}$-matrix $\mathbf{J}_{\mathbf{k}}^{\alpha}=\operatorname{diag}\left(\lambda_{k}^{\alpha}, \cdots, \lambda_{k}^{\alpha}\right)+\mathbf{H}_{\mathbf{k}}^{\alpha}$, where $r_{k} \times r_{k}$-matrix which first upper diagonal contains ones, all other its entries are zeros.

If matrix $\mathbf{A}_{\alpha}$ do not have repeated eigenvalues, then solution (20) can be presented in the form

$$
\mathbf{B}_{\alpha}=\mathbf{T}_{\alpha} \cdot\left(\operatorname{diag}\left(\exp \left(j \omega\left(x_{3}-x_{0}\right) \lambda_{1}^{\alpha}\right), \cdots, \exp \left(j \omega\left(x_{3}-x_{0}\right) \lambda_{6}^{\alpha}\right)\right)\right) \cdot \mathbf{T}_{\alpha}^{-\mathbf{1}} \cdot \mathbf{B}_{\alpha}^{\mathbf{0}}
$$

where $\mathbf{T}_{\alpha}$ is $6 \times 6$-matrix, the columns of which are eigenvectors of the matrix $\mathbf{A}_{\alpha}$. Applying to relations (11), (12), (7), (8) Fourier transform (15) we obtain

$$
\begin{gathered}
\left.\mathbf{B}_{\mathbf{I}^{\prime}}\right|_{x_{3}=x_{3}^{s}}-\left.\mathbf{B}_{\mathbf{I}^{\prime \prime}}\right|_{x_{3}=x_{3}^{s}}=\mathbf{F},\left.\quad \mathbf{B}_{\mathbf{I}^{\prime \prime}}\right|_{x_{3}=L-H}-\left.\mathbf{B}_{\mathbf{I I}}\right|_{x_{3}=L-H}=0, \\
\left.\mathbf{B}_{\mathbf{I I}}\right|_{x_{3}=L-H+h}-\left.\mathbf{B}_{\mathbf{I I I}}\right|_{x_{3}=L-H_{h}}=0,\left.\quad \mathbf{B}_{\mathbf{I I I}}\right|_{x_{3}=L}-\left.\mathbf{B}_{\mathbf{I V}}\right|_{x_{3}=L}=0,
\end{gathered}
$$

where

$$
\mathbf{F}=\left(\mathbf{U}_{\mathbf{s}}, \mathbf{S}_{\mathbf{s}}\right), \quad \mathbf{U}_{\mathbf{s}}=\left(U_{1}^{s}, U_{2}^{s}, U_{3}^{s}\right), \quad \mathbf{S}_{\mathbf{s}}=\left(S_{1}^{s}, S_{2}^{s}, S_{3}^{s}\right), \quad U_{i}^{s}=F\left(u_{s}^{s}\right), \quad S_{i}^{s}=F\left(\sigma_{s}^{s}\right) .
$$

The components $F_{\beta}, \beta=1,2, \ldots, 6$ of the source vector $\mathbf{F}$ can be expressed via the components $M_{\beta \gamma}$ of tensor of seismic moment $\mathbf{M}[7]$ :

$$
\begin{gathered}
F_{1}=-\frac{M_{13}}{\mu_{I}}, \quad F_{2}=-\frac{M_{23}}{\mu_{I}}, \quad F_{3}=-\frac{M_{33}}{\lambda_{I}+2 \mu_{I}}, \quad F_{4}=p_{1}\left(M_{11}-\frac{\lambda_{I}}{\lambda_{I}+2 \mu_{I}} M_{33}\right)+p_{2} M_{12}, \\
F_{5}=p_{1} M_{21}+p_{2}\left(M_{22}-\frac{\lambda_{I}}{\lambda_{I}+2 \mu_{I}} M_{33}\right), \quad F_{6}=p_{1}\left(M_{31}-M_{13}\right)+p_{2}\left(M_{32}-M_{23}\right) .
\end{gathered}
$$

Application of Fourier transform to the relations (5) yields

$$
\left.S_{I^{\prime}}\right|_{x_{3}=0}=0
$$

To find $\mathbf{B}_{\alpha}\left(x_{3}, p_{1}, p_{2}, \omega\right) \forall \alpha=I^{\prime}, I^{\prime \prime}, I I, \cdots, I V$, we should determine values of unknown vectors $\mathbf{B}_{\mathbf{I}^{\prime}}^{\mathbf{0}}$ to $\mathbf{B}_{\mathbf{I V}}^{\mathbf{0}}$. Each vector has 6 scalar constants. But because of radiation condition at $x_{3} \rightarrow \infty$ only three components of vector $\mathbf{B}_{\mathbf{I V}}^{\mathbf{0}}$ are independent. So, the whole number of scalar unknowns is 27. Substituting (20) into relations (23), we obtain 24 scalar equations. Relation (26) gives three equations. So, totally we have 27 equations.

To find components $u_{i}^{\alpha}\left(x_{1}, x_{2}, x_{3}, t\right)$ of displacement vector in the layers $\mathrm{七}_{\alpha}, \alpha=I^{\prime}, I^{\prime \prime}, I I, \cdots, I V$, we apply to corresponding $U_{1}^{\alpha}\left(x_{3}, p_{1}, p_{2}, \omega\right)$ the inverse Fourier transform

$$
u_{i}^{\alpha}=\mathcal{F}^{-1}\left(U_{i}^{\alpha}\right), \quad \mathcal{F}^{-1}(\cdots)=\frac{1}{8 \pi^{3}} \iiint(\cdots) \omega^{2} \mathrm{e}^{j \omega\left(t-p_{1} x_{1}-p_{2} x_{2}\right)} \mathrm{d} p_{1} \mathrm{~d} p_{2} \mathrm{~d} \omega .
$$

Further we use this method to study wave field interference of the free surface $x_{3}=0$. 


\section{Depth-velocity model of the structure}

We will consider the layers $\mathrm{E}_{I}$ and $\mathrm{E}_{I V}$ as massive (monolithic) elastic mediums with identical elastic properties. Suppose $\lambda$ and $\mu$ are known Lame constants of this layers. As to layers $\mathrm{E}_{I I}$ and $\mathrm{E}_{I I I}$, which are porous mediums, their properties are dependent on properties of materials they are built of, their porosity, properties of fluids they are saturated, and fluid's distributions in the porous spaces of the layers. So, to apply developed theory we need to determine elastic properties of porous layers $\mathrm{E}_{I I}$ and $\mathrm{E}_{I I I}$. To do this, we apply the theory of effective elastic moduli of porous media developed by Gassmann [2].

\section{Gassmann's model for effective elastic moduli of porous medium}

In concordance with Gassmann's model [2], the bulk $K$ and the shear $G$ moduli of porous media saturated with a fluid can be determined as

$$
K=K_{d r y}+\frac{\left(1-\frac{K_{d r y}}{K_{m}}\right)^{2}}{\frac{m}{K_{f}}+\frac{1-m}{K_{m}}-\frac{K_{d r y}}{K_{m}^{2}}}, \quad G=G_{d r y},
$$

where $K_{d r y}$ and $K_{m}$ are bulk moduli of the dry skeleton and of the solid substance what the skeleton is built of, $K_{f}$ stands for bulk modulus of the fluid, saturating the porous media, $m$ is porosity, and $G_{d r y}$ stands for shear modulus of dry skeleton.

The moduli of dry skeleton $K_{d r y}$ and $G_{d r y}$ of sandstone can be expressed via moduli $K_{m}$ and $G_{m}$ of the solid substance, what the skeleton is built of, and porositym [Goldberg and Gurevich, 1998, Carcione, 2000]:

$$
K_{d r y}=K_{m}(1-m)^{1+\frac{A}{1-m}}, \quad G_{d r y}=G_{m}(1-m)^{1+\frac{A}{1-m}} .
$$

Here $A$ is a dimensionless coefficient depending on geometry of the pore space and other skeleton's characteristics. In paper [14] parameter $A$ was evaluated between 2 and 4 .

When effective elastic moduli are known we can determine Lame coefficient for saturated porous medium [9]

$$
\lambda=K-\frac{2}{3} G, \quad \mu=G .
$$

The effective mass density $\rho$ of the saturated porous medium is determined as

$$
\rho=\rho_{m}(1-m)+\rho_{f} m
$$

where $\rho_{m}$ is the mass density of the solid substance, what the skeleton is built of, and $\rho_{f}$ is the mass density of the saturant.

Phase velocities of longitudinal $C_{p}$ and transversal $C_{s}$ elastic waves can be expressed via effective elastic moduli $K$ and $G$, and mass density $\rho[9]$ :

$$
C_{p}=\sqrt{\frac{\left(K+\frac{4}{3} G\right)}{\rho}}, \quad C_{s}=\sqrt{G / \rho} .
$$

To use the formulae (28)-(31) for calculation elastic property of layers $\mathrm{E}_{I I}$ and $\mathrm{E}_{I I I}$, we should determine distributions of density of the saturants in the layers and their bulk moduli. 


\section{Saturants distrubution in the porous layer}

In the equilibrium conditions distributions of the saturants in the layers $\mathrm{E}_{I I}$ and $\mathrm{七}_{I I I}$ are defined by the equations

$$
-\frac{\partial p_{g}}{\partial z}+g \rho_{g}=0, \quad-\frac{\partial p_{w}}{\partial z}+g \rho_{w}=0
$$

where $\rho_{g}$ and $\rho_{w}$ are the pressure and specific weight of gas in the layer $\mathrm{七}_{I I}, p_{w}$ and $\rho_{w}$ are the pressure and specific weight of water. The density the fluids $\rho_{g}$ and $\rho_{w}$ are dependent on their pressures:

$$
\rho_{g}=\frac{\mu_{g}}{Z\left(p_{1}, T\right) R T} p_{g}, \quad \rho_{w}=\rho_{0}\left(1+\frac{1}{\alpha_{w}}\left(p_{w}-p_{0}\right)\right) .
$$

Here $\mu_{g}$ is the molar mass of the gas, $T$ is absolute temperature, $Z\left(p_{g}, T\right)$ is the compressibility factor of gas that takes into account the departure of true gas from ideal gas behavior, $R$ stands for universal gas constant, $\alpha_{w}$ is the water's compressibility factor, $p_{0}$ stands for pressure on the ground-water level, $\rho_{0}$ is water's density at pressure $p_{0}$.

We take $Z\left(p_{1}, T\right)$ in the form

$$
Z\left(p_{g}, T\right)=Z\left(\bar{p}_{g}, \bar{T}\right) \equiv 1-A(\bar{T}) \bar{p}_{g}+B(\bar{T}) \bar{p}_{g}^{2}
$$

where $A(\bar{T})=3.52 \exp (-2.260 \bar{T}), B(\bar{T})=0.274 \exp (-1.878 \bar{T}), \bar{p}_{g}=p_{g} / p_{c}, \bar{T}=T / T_{c}, p_{c}$ and $T_{c}$ are critical pressure and temperature of the gas.

If to eliminate variables $\rho_{g}, \rho_{w}$ from equations (33) using relations (34), we obtain

$$
\begin{gathered}
\frac{\partial \bar{p}_{g}}{\partial \zeta}-\frac{1}{Z\left(\bar{p}_{1}, \bar{T}\right)} \bar{p}_{g}=0, \quad 0<\zeta<\bar{h}, \\
\frac{\bar{p}_{w}-\bar{p}_{0}}{\partial \zeta}-\frac{z_{0}}{z_{1}}\left(\bar{p}_{w}-\bar{p}_{0}\right)=\bar{\rho}_{0}, \quad \bar{h}<\zeta<\bar{H} .
\end{gathered}
$$

Here we have introduced the dimensionless coordinate $\zeta=\left(x_{3}-L+H\right) / z_{0}$, where $z_{0}=R T /\left(g \mu_{g}\right)$, and used the denotations $\bar{h}=h / z_{0}, \bar{H}=H / z_{0}, z_{1}=\alpha_{w} /\left(g \rho_{0}\right), \bar{p}_{0}=p_{0} / p_{c}, \bar{\rho}_{0}=\rho_{0} / \rho_{c}, \rho_{c}=$ $\mu_{g} p_{c} /(R T)$.

As the pressure on the bottom $\zeta=\bar{H}$ of the porous stratum is formed by water head of height $L$, we have

$$
\left.\bar{p}_{w}\right|_{\zeta=\bar{H}}=\bar{\rho}_{0} \frac{z_{1}}{z_{0}}\left(\exp \left(\frac{z_{0}}{z_{1}} \bar{L}\right)-1\right)+\bar{p}_{0} .
$$

On the boundary between the layers $\mathrm{E}_{I I}$ and $\mathrm{E}_{I I I}$ we have the condition

$$
\left.\bar{p}_{g}\right|_{\zeta=\bar{H}}=\left.\bar{p}_{w}\right|_{\zeta=\bar{H}} .
$$

(36b) is a linear equation. Integrating it with boundary conditions (37), we obtain

$$
\bar{p}_{w}(\zeta)=\bar{\rho}_{0} \frac{z_{1}}{z_{0}}\left(\exp \left(\frac{z_{0}}{z_{1}} \zeta+\bar{L}-\bar{H}\right)-1\right)+\bar{p}_{0}, \quad \bar{h}<\zeta<\bar{H}
$$

The formula (39) determines pressure of water in the layer $\mathrm{E}_{I I I}$. Using it we can determine the right hand side in (38)

$$
\left.\bar{p}_{w}\right|_{\zeta=\bar{H}}=\bar{p}_{w}(\bar{h})=\bar{\rho}_{0} \frac{z_{1}}{z_{0}}\left(\exp \left(\frac{z_{0}}{z_{1}} \bar{L}+\bar{h}-\bar{H}\right)-1\right)+\bar{p}_{0}
$$

and obtain boundary condition for (36a). 
(36a) is a separable equation. Integrating it with accounting (38), (40), we obtain

$$
\begin{aligned}
\ln \frac{p_{g}(\zeta)}{p_{w}(\zeta)}-A(\bar{T})\left(p_{g}(\zeta)\right. & \left.-p_{w}(\zeta)\right) \\
& +\frac{1}{2} B(\bar{T})\left(p_{g}(\zeta)^{2}-p_{w}(\zeta)^{2}\right)+C(\bar{T})(\zeta-\bar{h}) / \bar{T}=0, \quad 0 \leqslant \zeta \leqslant \bar{h}
\end{aligned}
$$

where $C(\bar{T}) \equiv 1-A(\bar{T})+B(\bar{T})$.

Solving nonlinear equation (41) with respect to $p_{g}(\zeta)$ for each $\zeta \in(0, \bar{h})$, we can determine gas pressure in the layer $\mathrm{E}_{I I}$ as a function of its thickness coordinate.

To determine the densities $p_{g}(\zeta)$ and $p_{w}(\zeta)$ of the fluids in layers $\mathrm{七}_{I I}$ and $\mathrm{七}_{I I I}$ we use the equations of state (34).

It should be stressed that, when parameters $\bar{L}$ and $\bar{H}$ are given, the function $p_{g}(\zeta)$, determining by solving equation (9), is depended on parameter $\bar{h}: p_{g}=p_{g}(\zeta, \bar{h})$, which, in turn, is dependent on the amount of the gas accumulated in the layer.

Let $M\left[\mathrm{~kg} / \mathrm{m}^{2}\right]$ be the mass of the gas accumulated in the porous space of $\mathrm{E}_{I I}$ referred to unit area of its middle surface, and $m$ is the porosity of the layer. Then we have the relation

$$
\int_{0}^{\bar{h}} p_{g}(\zeta, \bar{h}) \mathrm{d} \zeta=\frac{1}{m} \bar{M}
$$

where $\bar{M}=M / z_{0}$.

The relation (42) establishes interdependence between the amount $M$ of gas accumulated in the layer $\mathrm{E}_{I I}$ on the one hand and its thickness $h$, thickness $H$ of the whole porous layer $\mathrm{E}=L_{I I} \bigcup \mathrm{七}_{I I I}$ and its depth $L$ on the other hand.

Solving the equation (42) with respect to $\bar{h}$ at fixed $H$ and $L$, we can determine the thickness $h$ of the layer $\mathrm{E}_{I I}$, saturated with gas, depending on the amount of the gas accumulated in it.

Mass capacity of the layer is determined by its surface mass density that is attained at $h=H$ (when the whole porous layer $L$ is saturated with gas):

$$
\bar{M}_{\max }=m \int_{0}^{\bar{H}} p_{g}(\zeta, \bar{H}) \mathrm{d} \zeta .
$$

Fig. 1 illustrates how porous pressure $\bar{p}(\zeta)$ in the layer $\mathrm{E}$ changes depending on amount of gas stored in the stratum. We define $\bar{p}(\zeta)$ as

$$
\bar{p}(\zeta)= \begin{cases}p_{g}(\zeta), & 0<\zeta<\bar{h}, \\ p_{w}(\zeta), & \bar{h}<\zeta<\bar{H} .\end{cases}
$$

Curves 1, 2 and 3 in the figure correspond to different values of dimensionless parameter: $\bar{h}=0.01,0.02$ and 0.03 .

The calculations were made at temperature $T=340 \mathrm{~K}$ for layer Ł with dimensionless thickness $\bar{H}=0.03$ and depth $\bar{L}=0.3$. The following values for parameters were taken for gas: $\mu=16.043, p_{c}=$ $4.649 \mathrm{MPa}, T_{c}=190.9 \mathrm{~K}$, characteristic length was $z_{0} \approx 2926.35 \mathrm{~m}$. For water we took $\rho_{0}=10^{3} \mathrm{~kg} / \mathrm{m}^{3}, p_{0}=10^{5} \mathrm{~Pa}, \alpha_{w}=2.4 \mathrm{GPa}$, characteristic length was $z_{1}=2 / 45 \cdot 10^{5} \mathrm{~m}$.

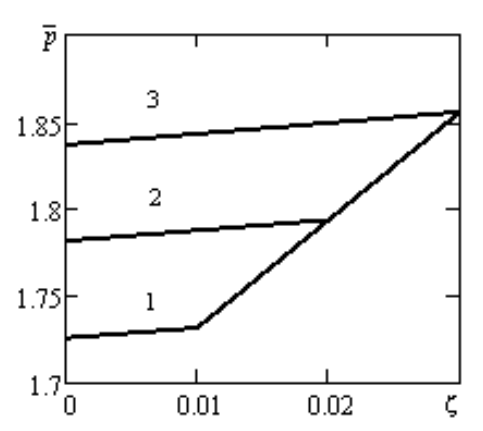

Fig. 1. Distribution of porous pressure in the layer $\mathrm{E}$.

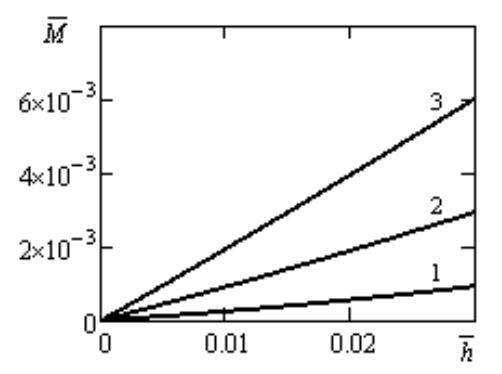

Fig. 2. Dependence between the amount of accumulated gas and the thickness $h$, of the layer $\mathrm{E}_{I I}$ for porous layer $\mathrm{E}$ of thickness $\bar{H}=0.03$. 
As we can see the pore pressure changes in the layer $\mathrm{E}$ on about $8 \%$.

Fig. 2 shows how the boundary dividing the part of the layer $\mathrm{E}$ saturated with gas and other one saturated with water is moving when the amount of stored gas is varying. Curves 1, 2 and 3 correspond to different values of depth $\bar{L}=0.1,0.3$ and 0.6 correspondingly.

\section{Stadying the effective elastic moduli and phase velocities of plane waves in the porous layer}

Now we can define the bulk modulus $K_{f}$ for fluid which appears in the formula (28) for effective bulk modulus of the saturated porous medium.

Bulk modulus for gas is defined as

$$
\alpha_{g}=\rho_{g} \frac{\partial p_{g}}{\partial \rho g} .
$$

Applying (44) to the state equation (34)1 and using relation (33) we obtain

$$
\alpha_{g}=\frac{Z\left(\overline{p_{g}}, \bar{T}\right) p_{g}}{\left(1-B(\bar{T}){\overline{p_{g}}}^{2}\right)} .
$$

As we can see bulk modulus of gas increases with it pressure. Unlike gas, the compressibility of water almost doesn't depend on the pressure. We can take it constant $\alpha_{w}$ (see equation $\left.(34)_{2}\right)$.

So the bulk modulus of the fluid $K_{f}$ in the layer $\mathrm{E}$ is dependent on the coordinate $\zeta \in(0, \bar{H})$ and defined by the piecewise continuous function with the point of discontinuity $\zeta=\bar{H}$ :

$$
K_{f}(\zeta)= \begin{cases}\alpha_{g}(\zeta), & 0<\zeta<\bar{h}, \\ \alpha_{w}(\zeta), & \bar{h}<\zeta<\bar{H}\end{cases}
$$

where $\alpha_{g}(\zeta)$ is defined according to (45). With the use (34), (35), (39), (41) we can calculate, applying the formula (31), the effective mass density $\rho$ of the saturated porous medium. Value $\rho_{f}$ in this formula is changing along the transversal coordinate $\zeta$ and can be defined by the piecewise continuous function with the point of discontinuity $\zeta=\bar{h}$ :

$$
\rho_{f}(\zeta)= \begin{cases}\rho_{g}(\zeta), & 0<\zeta<\bar{h} \\ \rho_{w}(\zeta), & \bar{h}<\zeta<\bar{H}\end{cases}
$$

Fig. 3 shows dependence of effective mass density of the saturated porous layer on the coordinate $\zeta$ at two different values of gas amount stored in it: a) $-\bar{h}=0.01$ and b) $-\bar{h}=0.02$.

The layer's thickness and depth are $\bar{H}=0.03$ and $\bar{L}=0.3$.
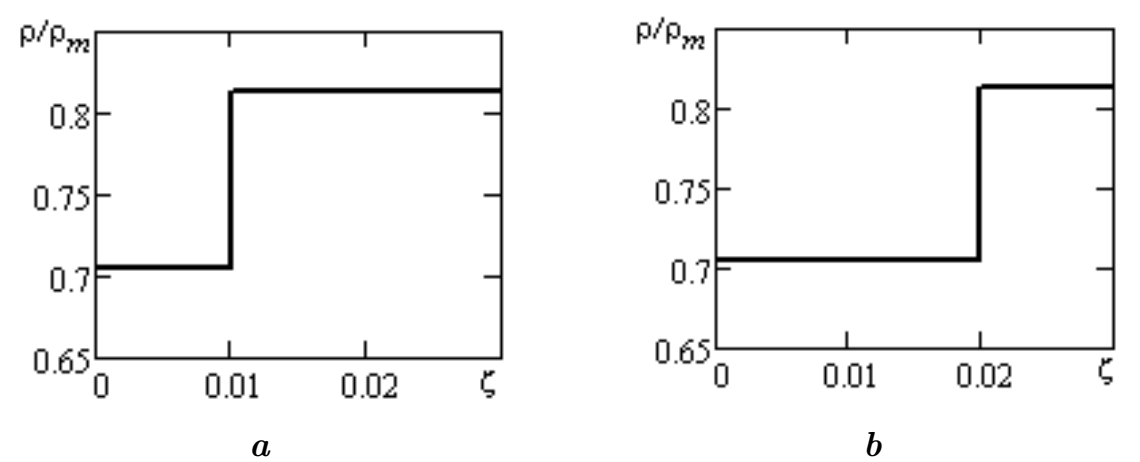

Fig. 3. Dependence of the effective mass density reffered to $\rho_{m}$ on coordinate $=\zeta$ at two values of $\bar{h}=0.01,0.02$. 
So, as we can see, in spite of that $\rho_{f}$ is changing in $\mathrm{L}$ on about $8 \%$, layer $\mathrm{E}$ itself can be considered as piece-wise homogeneous one with respect to its effective mass density. With the use (34), (35), (39), (41), (45), (46) we can calculate, applying formulas (28), (29) the effective elastic moduli $K$ and $G$ of saturated porous layer as function of its transversal coordinate depending on amount of the gas accumulated in the layer, and parameters $L$ and $H$. As the effective modulus $K$ is depended on bulk modulus of fluid, its value is changing with the thickness coordinate $\zeta$. It can be represented by a piecewise continuous function with the point of discontinuity $\zeta=\bar{h}$. Applying formulas (32) we can calculate the phase velocities of longitudinal $C_{P}$ and transversal $C_{S}$ elastic waves in the layer $\mathrm{E}$.

Figs. 4 and 5 show the dependences of the phase velocities of longitudinal $C_{P}$ and transversal $C_{S}$ acoustic waves in the saturated porous layer $\mathrm{E}$ on the coordinate $\zeta$ at two different values of gas amount stored in it: a) $-\bar{h}=0.01$ and b) $-\bar{h}=0.02$. The layer's $\mathrm{E}$ thickness and depth are $\bar{H}=0.03$ and $\bar{L}=0.3$.
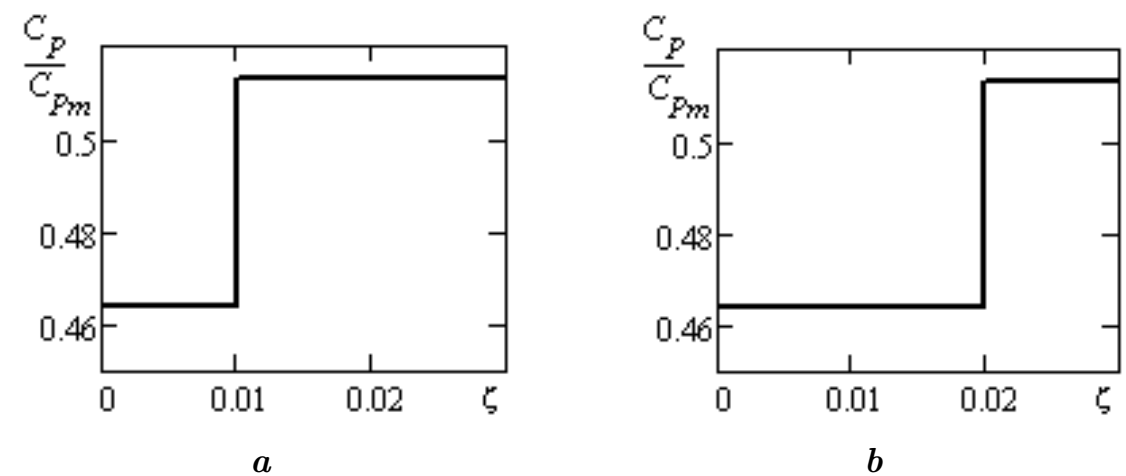

Fig. 4. Longitudinal wave phase velocity in the porous layer at two values of $\bar{h}=0.01,0.02$.

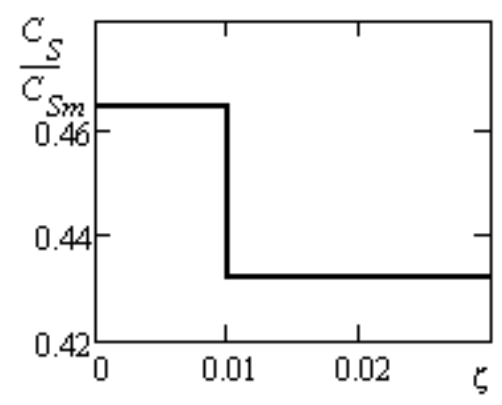

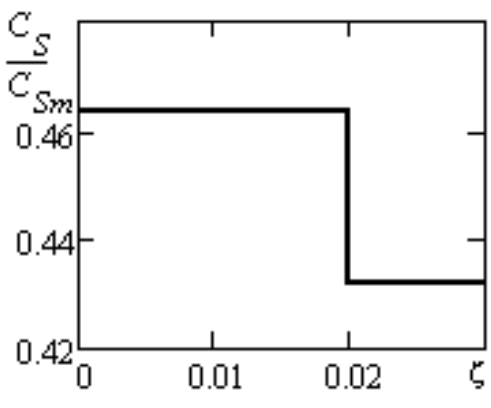

$b$

Fig. 5. Transversal wave phase velocity in the porous layer at two values of $\bar{h}=0.01,0.02$.

The calculations presented in this section were made under the next values of parameters: $K_{m}=$ $3.9 \cdot 10^{10} \mathrm{~Pa}, G_{m}=3.3 \cdot 10^{10} \mathrm{~Pa}, \rho_{m}=2650 \mathrm{~kg} / \mathrm{m}^{3}, A=3$.

As we can see the layer $L$ itself can be considered as piece-wise homogeneous one with respect to phase velocities of longitudinal and transversal elastic plane waves.

So, in this section we calculated elastic properties of porous layer, saturated with gas and water, and justified possibility to apply the model of piece-wise homogeneous elastic layered structure for describing elastic waves interaction with the layer.

\section{Studying wave interference patterns on the free surface}

We applied the approach developed in the sections 2 and 3 to study a wave interference pattern on free surface of the layered structure. We also interested how the pattern is changing with variation the amount of gas accumulated in the porous layer. 
The calculations were made for depth $L=1000 \mathrm{~m}$ and thickness $H=200 \mathrm{~m}$ of the layer $\mathrm{E}$. The properties of the layers $\mathrm{L}_{I}$ to $\mathrm{L}_{I V}$, which have been used in calculations, are presented in the table 1 .

Table 1. The properties of the layers.

\begin{tabular}{|l|c|c|c|c|}
\hline Layer & $\mathrm{Ł}_{I}$ & $\mathrm{Ł}_{I I}$ & $\mathrm{Ł}_{I I I}$ & $\mathrm{Ł}_{I V}$ \\
\hline$\lambda, \mathrm{kg} /\left(\mathrm{s}^{2} \mathrm{~m}\right), \cdot 10^{10}$ & 8.298 & 1.279 & 1.776 & 8.298 \\
\hline$\mu, \mathrm{kg} /\left(\mathrm{s}^{2} \mathrm{~m}\right), \cdot 10^{10}$ & 3.300 & 0.514 & 0.500 & 3.300 \\
\hline$\rho, \mathrm{kg} / \mathrm{m}^{3}$ & 2650 & 1887 & 2156 & 2650 \\
\hline$C_{P}, \mathrm{~m} / \mathrm{s}$ & 5596 & 2593 & 2870 & 5596 \\
\hline$C_{S}, \mathrm{~m} / \mathrm{s}$ & 3529 & 1630 & 1524 & 3529 \\
\hline
\end{tabular}

The pulse source of uniform extension was situated at the point $S$ with coordinates $x_{1}=0$, $x_{2}=0, x_{3}^{s}=250 \mathrm{~m}$. Its seismic moment was determined as $M_{i i}=M_{0} \varphi(t), M_{i j}=0, i \neq j$, where $M_{0}=9.3 \cdot 10^{16} \mathrm{Nm}, \varphi(t)=\exp \left(t^{2} / \tau_{0}^{2}\right), \tau=0.1 \mathrm{~s}$.

To study how the wave-field pattern varies, when thickness $h$ of the sublayer saturated with gas is changing, we solved the problem several times - each time at another value of thickness $h$. Let $u_{i}^{I^{\prime}}\left(x_{1}, x_{2}, x_{3}, t ; h\right)$ be wave field in layer $\mathrm{E}_{I^{\prime}}$ calculated for case, when the thickness of the layer $\mathrm{E}_{I I}$ is equal $h$, and $P$ be a point on the free surface (point of sight) with coordinate $x_{1}=x_{1}^{P}, x_{2}=$ $x_{3}=0$. Time dependences of tangential $U_{1}=(t, h)=u_{1}^{I^{\prime}}\left(x_{1}^{P}, 0,0, t ; h\right)$ and normal $U_{3}=(t, h)=$ $u_{3}^{I^{\prime}}\left(x_{1}^{P}, 0,0, t ; h\right)$ components of displacement vector at the point $P$ with coordinates $x_{1}^{P}=2000 \mathrm{~m}$, $x_{2}=0, x_{3}=0$ are shown on the figure 6 and 7 . Each curve on the figures corresponds to specific value of thickness $h$ of the layer $\mathrm{E}_{I I}$.

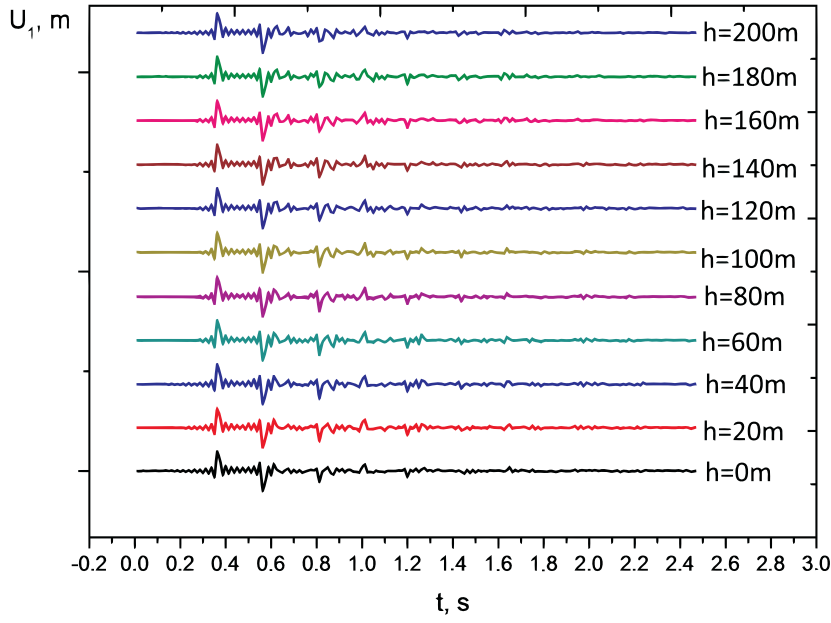

Fig. 6. The time dependences of the tangential component of displacement vector at point of sight on the free surface for different values of thickness $h$ of the layer $\mathrm{E}_{I I}$.

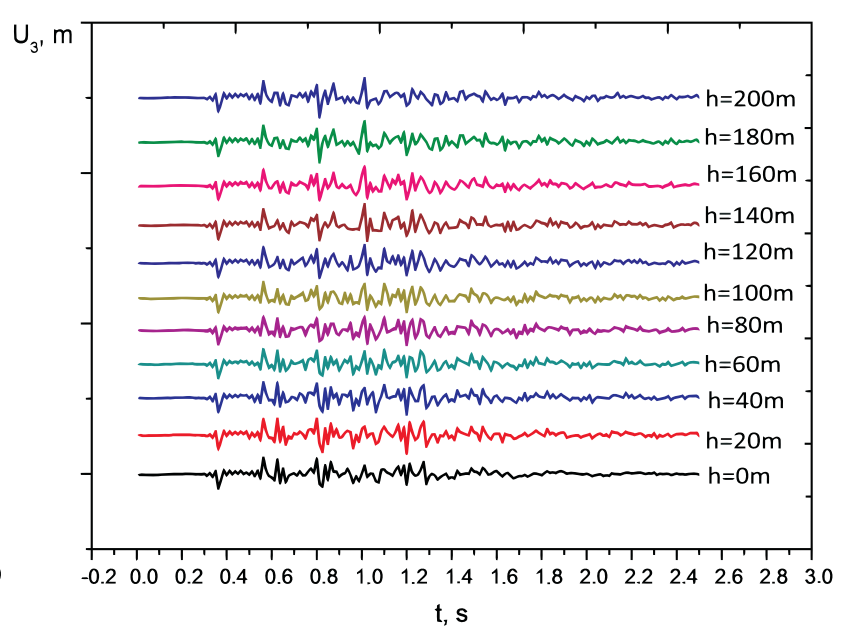

Fig. 7. The time dependences of the normal component of displacement vector at point of sight on the free surface for different values of thickness $h$ of the layer $\mathrm{Ł}_{I I}$.

As we can see, within the interval $(0.3 \ldots 0.6)$ s all curves are identical - they do not change with $h$. The first two peaks on the curves correspond to $P$ - and $S$-waves which came to the point $P$ directly from the source. The next peak at $t \neq 0.8 \mathrm{~s}$ correspond to a wave reflected by the boundary between $\mathrm{E}_{I}$ and $\mathrm{E}_{I I}$. It doesn't move along time axis with changing $h$, but its shape and amplitude change with $h$. Next peaks correspond waves reflected by boundaries between layers $\mathrm{七}_{I I}$ and $\mathrm{七}_{I I I}$ and between $\mathrm{七}_{I I I}$ and $\mathrm{七}_{I V}$.

We can receive evidence of this by comparing the curves of figures 6 and 7 with those shown on the figures 8 and 9 correspondingly. There the time dependences of tangential and normal displacements 
at the point of sight are shown on these figures. The curves 1 on the figures 8 and 9 were calculated for two-layered structure consisting of bulk layer $\mathrm{E}_{I}$ and porous half-space saturated with gas. The curves 2 on these figures were calculated for three-layered structure consisting of bulk layer $\mathrm{E}_{I}$, porous layer of thickness $H$, saturated with gas, and porous half-space, saturated with water.

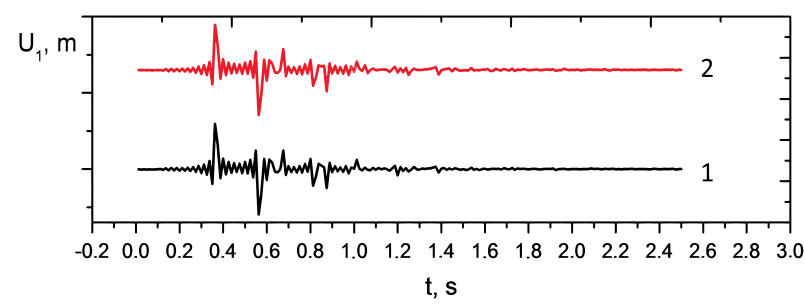

Fig. 8. The time dependences of the tangential components of displacement vector at the point of sight on the free surface for two-layered structure (1) and three-layered structure (2).

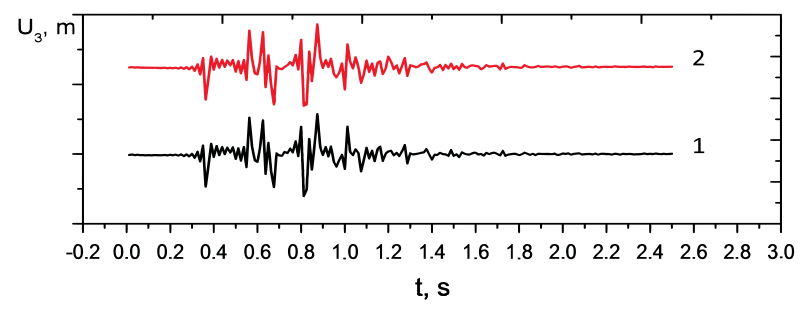

Fig. 9. The time dependences of the normal components of displacement vector at the point of sight on the free surface for two-layered structure (1) and threelayered structure (2).

Analyzing the time dependences, shown on the figures 6 and 7 , we can conclude, that at $t>0.6$ the curves, calculated for different thickness $h$, are differ. So, the curves contain information about the structure of porous layer $\mathrm{L}$.

To establish a quantitative relationship between thickness $h$ and the time dependences of displacements $U_{i}(t, h)$ of free surface, calculated at this $h$, we used correlation coefficients $R_{U_{i}}^{w}$ and $R_{U_{i}}^{g}$, $i=1,2,3$ :

$$
\begin{aligned}
R_{U_{i}}^{w}= & \frac{\int_{t_{1}}^{t_{2}}\left(U_{i}(t, h)-\bar{U}_{i}(h)\right)\left(U_{i}(t, 0)-\bar{U}_{i}^{0}\right) \mathrm{d} t}{\sqrt{\left(U_{i}(t, h)-\bar{U}_{i}(h)\right)^{2} \mathrm{~d} t} \sqrt{\left(U_{i}(t, 0)-\bar{U}_{i}^{0}\right)^{2} \mathrm{~d} t}}, \\
R_{U_{i}}^{g}= & \frac{\int_{t_{1}}^{t_{2}}\left(U_{i}(t, h)-\bar{U}_{i}(h)\right)\left(U_{i}(t, H)-\overline{U_{i}^{H}}\right) \mathrm{d} t}{\sqrt{\left(U_{i}(t, h)-\bar{U}_{i}(h)\right)^{2} \mathrm{~d} t} \sqrt{\left(U_{i}(t, H)-\bar{U}_{i}^{H}\right)^{2} \mathrm{~d} t}} .
\end{aligned}
$$

Here

$$
\bar{U}_{i}(h)=\frac{1}{t_{2}-t_{1}} \int_{t_{1}}^{t_{2}} U_{i}(t, h) \mathrm{d} t, \quad \bar{U}_{i}^{0}=\frac{1}{t_{2}-t_{1}} \int_{t_{1}}^{t_{2}} U_{i}(t, 0) \mathrm{d} t, \quad \bar{U}_{i}^{H}=\frac{1}{t_{2}-t_{1}} \int_{t_{1}}^{t_{2}} U_{i}(t, H) \mathrm{d} t .
$$

Measure $R_{U_{i}}^{w}(h)$ define deviation of curve $U_{i}(t, h)$ from the curve $U_{i}(t, 0)$ which corresponds the case when the whole porous layer $\mathrm{E}$ has been saturated with water (the storage facility is empty). Measure $R_{U_{i}}^{g}(h)$ define deviation of curve $U_{i}(t, h)$ from the curve $U_{i}(t, H)$, which corresponds the case when the whole porous layer $\mathrm{E}$ has been saturated with gas (the storage facility is empty).

On Figs. 10 the dependences of introduced measures $R_{U_{i}}^{w}(h)$ and $R_{U_{i}}^{g}(h)$ for tangential $(i=1)$ and transversal $(i=3)$ displacements are presented. Different curves on the plots correspond to different ranges of integration, which have been used in formula $(47):(0 \ldots 2.5) \mathrm{s},(0.6 \ldots 2.5) \mathrm{s}$, and $(0.6 \ldots 1.6) \mathrm{s}$. The curves $R_{U_{i}}^{w}(h)$ and $R_{U_{i}}^{g}(h)$ corresponding to these time intervals are marked on the plots by squares, triangles and circles respectively.

As we can see there one-to-one correspondences exist between $R_{U_{i}}^{w}(h)$ and $h$ as well as between $R_{U_{i}}^{g}(h)$ and $h$. This means that if we know $R_{U_{i}}^{w}(h)$ or $R_{U_{i}}^{g}(h)$ we can determine $h$. It was shown in 

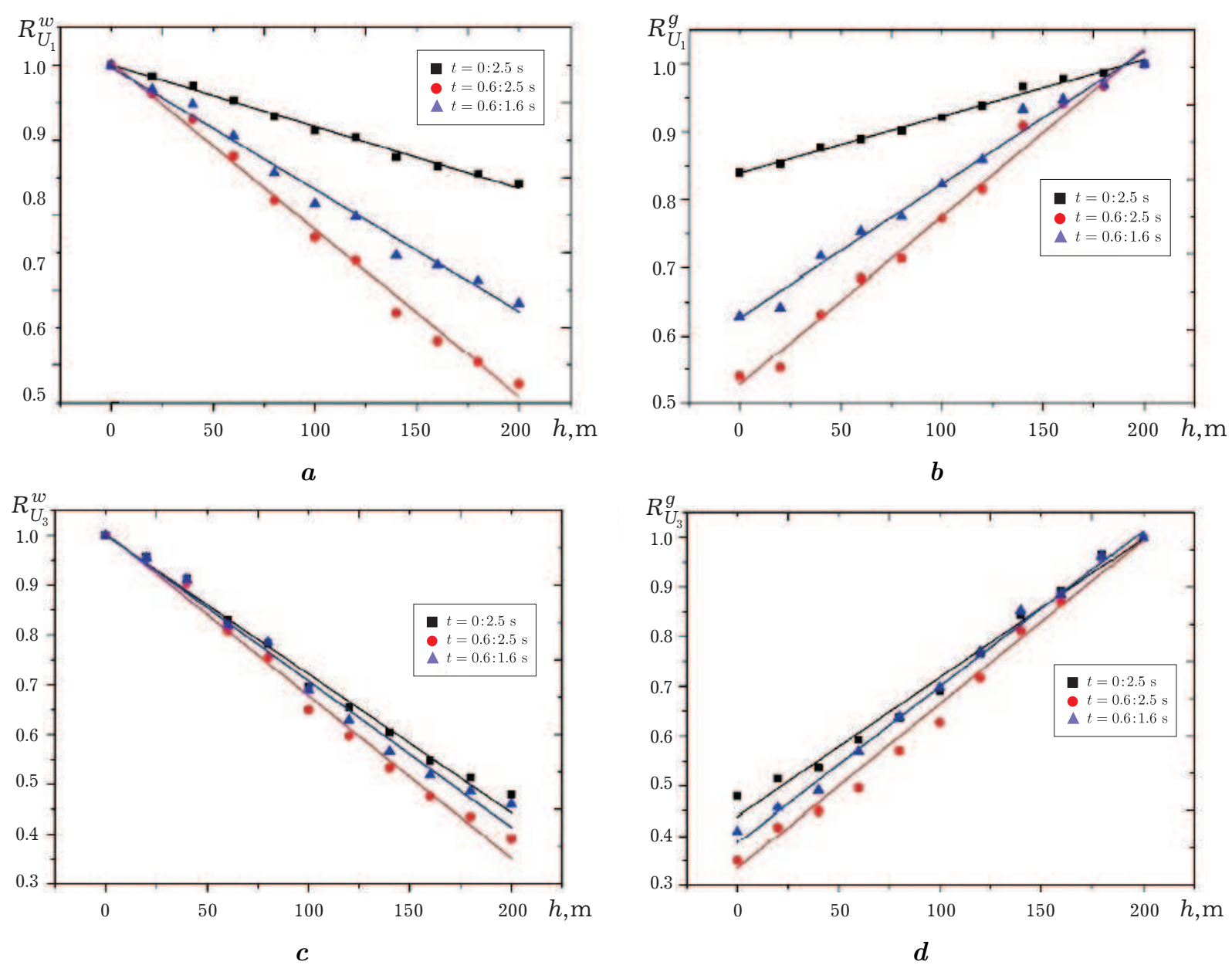

Fig. 10. Dependeces of the measures $R_{i}^{w}, R_{i}^{g}$ on thickness $h$ of the layer $\mathrm{七}_{I I}$.

section IV, that one-to-one correspondence exists between $\mathrm{h}$ and amount $M$ of gas accumulated in the porous layer (see Fig. 2). So, we can also determine $M$.

We can see that the measures $R_{U_{i}}^{w}(h)$ and $R_{U_{i}}^{g}(h)$ calculated for interval $(0 \ldots 2.5)$ s possess the greatest self-descriptiveness because the slopes of the lines calculated for this time interval are the greatest. We can also conclude that measures $R_{U_{i}}^{w}(h)$ and $R_{U_{i}}^{g}(h)$, calculated for transversal displacement $U_{3}(t, h)$, are more informative as compared to those $R_{U_{i}}^{w}(h)$ and $R_{U_{i}}^{g}(h)$, calculated for tangential displacement $U_{1}(t, h)$. This appears from the fact that $R_{U_{i}}^{w}(h)$ and $R_{U_{i}}^{g}(h)$ are varied between 1.0 and 0.35 , when $h$ changes in interval $[0, H]$, whereas $R_{U_{i}}^{w}(h)$ and $R_{U_{i}}^{g}(h)$ are varied between 1.0 and 0.55 .

\section{Conclusions}

Underground gas storage facilities, which are built in aquifer, can be considered as a layered structure containing a porous layer $\mathrm{E}$ saturated partly with gas and partly with water. The boundary, which divides these two parts, is displacing when amount of the gas stored in the layer is changing. The analytical relations establishing distributions of gas and water in porous space of the layer $€$ have been obtained. On this basis the direct relationship between the amount of gas accumulated in porous layer and thickness $h$ of the sublayer saturated with gas, has been established.

With the use of the relations, determining distributions of fluids in the porous space, and of Grassmann's model for dynamics of porous media, the effective elastic moduli and the phase velocities of elastic waves in the sublayers saturated with gas and water have been calculated. As a result it was established that layer $\mathrm{E}$, saturated with the two fluids (gas and water), can be considered as a piece-wise homogeneous structure. 
In concordance with this, the semi-infinite horizontally-layered elastic structure containing a porous layer, saturated partly with gas and partly with water, has been considered as a model of the underground gas storage facility. The mathematical model describing interaction of elastic waves excited in the structure by a point pulse source, situated in the layer covering the porous layer $\mathrm{E}$, has been built and corresponding boundary-value problem formulated.

The problem was being solved with the use of Fourier transform. Time dependences of tangential and normal displacements in the point of sight, situated on the free structure's surface, have been studied depending on the amount of gas accumulated in the layer.

Direct relationship between the thickness $h$ of sublayer, saturated with gas, and wave-field pattern, calculated for this thickness $h$, has been established. Numeric measures which establish a relation between the thickness $h$ and calculated at this $h$ time dependences have been introduced. It was shown that these measures are almost linearly depend on $h$. This result can be useful for development of distant methods for operative a posteriori determination of amounts of natural gas accumulated in underground storage facilities.

[1] Underground Storage of Natural Gas, in Encyclopeadia of Hydrocarbons, vol. 1/ Exploration, Production and Transport. Rome: Eni. Istituto della Enciclopedia italiana (2005).

[2] Gassmann F. Elastic waves through a packing of sferes. Geophysics. 16 (4), 673-685 (1951).

[3] Sun W., Ba J., Carcione J. M. Theory of wave propagation in partially saturated double-porosity rocks: a triple-layer patchy model. Geophys. J. Int. 205 (1), 22-37 (2016).

[4] Carcione J. M. Wave Fields in Real Media: Wave Propagation in Anisotropic, Anelastic, Porous and Electromagnetic Media. 3rd edn. Elsevier Science (2015), 690 p.

[5] Rubino J. G., Holliger K. Seismic attenuation and velocity dispersion in heterogeneous partially saturated porous rocks. Geophys. J. Int. 188, 1088-1102 (2012).

[6] Aki K., Richards P. G. Quantitative Seismology. 2-nd Edition W. H. Freeman and Company (2002), 700 p.

[7] Fryer G. J., Frazer L. N. Seismic waves in stratified anisotropic media. Geophys. J. Roy. and Soc. 78 (4), 691-710 (1984).

[8] Fryer G. J., Frazer L. N. Seismic waves in stratified anisotropic media. II. Elastodynamic eigensolutions for some anisotropic systems. Geophys. J. Roy. and Soc. 91 (4), 73-101 (1987).

[9] Ba J., Carcione J. M., Sun W. T. Seismic attenuation due to heterogeneities of rock fabric and fluid distribution. Geophys. J. Int. 202 (3), 1843-1847 (2015).

[10] Ba J., Carcione J. M., Nie J.X. Biot-Rayleigh theory of wave propagation in double-porosity media. J. geophys. Res. 116, 1-12 (2011).

[11] Sona M. S., Kangb Y. J. Propagation of shear waves in a poroelastic layer constrained between two elastic layers. Applied Mathematical Modelling. 36 (8), 3685-3695 (2012).

[12] Hirsh M. W., Smale S., Devaney R. L. Differential equations, dynamical systems \& Chaos. Academic press (2004), $418 \mathrm{p}$.

[13] Quintal B., Steeb H., Frehner M., Schmalholz S. M. Quasi-static finite element modeling of seismic attenuation and dispersion due to wave-induced fluid flow in poroelastic media. J. geophys. Res.: Solid Earth. 116, 1-17 (2011).

[14] Boruah N., Chatterjee R. Rock physics template (RPT) analysis of well logs and seismic data for lithology and fluid classifications. Petroview. 3, 1-8 (2010). 


\title{
Математичне моделювання поширення пружних збурень у структурі, яка містить пористий шар, насичений газом і водою
}

\author{
Чекурін В..$^{1,2}$, Павлова А. Ю. ${ }^{3}$ \\ ${ }^{1}$ Інститут прикладних проблем механіки і математики ім. Я. С. Підстригача НАН Украйни \\ вул. Наукова, 3-б, 79060, Львів, Украӥна \\ ${ }^{2}$ Куявсько-Поморський університет, вул. Торунсъка, 55-57, 85-023, Бидгощ, Польща \\ ${ }^{3}$ Карпатсъке відділення інституту геобізики ім. С. І. Субботіна НАН України \\ вул. Наукова, 3-б, 79060, Львів, Украӥна
}

\begin{abstract}
Досліджено взаємодію пружних хвиль, зумовлених локальним імпульсним джерелом, із півбезмежною горизонтально-шаруватою структурою, що містить пористий шар, насичений газом і водою. Запропоновано математичну модель, яка описує розподіл газу і води в поровому просторі шару залежно від кількості накопиченого в ньому газу. В межах моделі обчислені ефективні пружні модулі областей пористого шару, насичених газом і водою. Сформульовано початково-крайову задачу динаміки пружних хвиль у структурі та із застосуванням перетворення Фур'є отримано $\dddot{11 ̈ ~ р о з в ' я з о к . ~ І з ~}$ використанням отриманого розв'язку досліджено картину хвильового поля на вільній поверхні структури залежно від кількості газу, нагромадженого в пористому шарі. Введено та чисельно досліджено характеристики, які пов'язують параметри хвильового поля на вільній поверхні із кількістю газу, нагромадженого в пористому шарі. Отримані результати можна застосувати для розроблення дистанційних методів обліку кількості природного газу в підземних сховищах газу, споруджених у водоносних горизонтах.
\end{abstract}

Ключові слова: шаруваті структури; пористі середовища; пружні збурення.

2000 MSC: $74 \mathrm{~J} 20$

удК: 550.344 